\title{
Wavelet-based Denoising of Partial Discharge Signals Buried in Excessive Noise and Interference
}

\author{
L. Satish and B. Nazneen \\ Dept. of High Voltage Engg. \\ Indian Institute of Science \\ Bangalore 560012, India
}

\begin{abstract}
Achieving acceptable levels of sensitivity during on-line and/or on-site partial discharge (PD) measurements still continues to remain a very challenging task, primarily due to strong coupling of external (random, discrete spectral and stochastic pulsive) interferences. Many analog and digital approaches have been proposed for suppressing these interferences, and amongst these, rejection of the pulsive type of interferences is known to be very difficult, if not impossible. The time and frequency characteristics of the pulsive interference being very similar to that of the PD pulses is the main reason posing difficulty in their separation. In this paper, a novel, semi-automatic, and empirical wavelet-based method (using multi-resolution signal analysis) is proposed to recover PD pulses, buried in excessive noise/interference comprising of random, discrete spectral, pulsive, and any combination of these interferences occurring simultaneously and overlapping-in-time with the PD pulses. A critical assessment of the proposed method is carried out, by processing both simulated and practically acquired PD signals. The results obtained are also compared with those from the best digital filter (infinite impulse response, IIR and finite impulse response, FIR) method proposed in literature. From the results it emerges that, the wavelet approach is superior and further, has the unique capability of successfully rejecting all the three kinds of interferences, even when PD signals and one or all interferences occur simultaneously and overlap-in-time.
\end{abstract}

Index Terms - Wavelets, multi-resolution signal analysis, partial discharges, PD signals, discrete spectral and pulsive interferences, PD signal denoising, digital filtering, on-site PD measurements, diagnostics and monitoring.

\section{INTRODUCTION}

$\mathrm{E}$ LECTRICAL insulation constitutes a very critical component of HV power apparatus. In spite of advances in the areas of manufacturing, processing, optimal design and quality control, these apparatus have continued to fail while in service. Investigations reveal that, in most cases the insulation is the primary cause. In view of this, power utilities are increasingly resorting to diagnostic measurements to assess the status of the insulation system. Amongst others, PD measurements have emerged as an indispensable, non-destructive, sensitive and powerful diagnostic tool. Using this diagnostic data, an overall assessment of the insulation system is possible. With the advent of digital PD techniques, these measurements are nowadays being performed on-site, and sometimes, on-line as well. A major bottleneck encountered with such measurements is the ingress of external. inter-

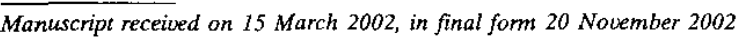

ferences (usually of very high amplitude comparable to PD signal) that directly affects the sensitivity and reliability of the acquired PD data. Major external interferences encountered during on-site PD measurements and their sources are:

- Discrete spectral interferences (DSI) from radio transmissions and power line carrier communication systems.

- Periodic pulse shaped interferences from power electronics or other periodic switching etc.

- Stochastic pulse shaped interferences from infrequent switching operations or lightning, arcing between adjacent metallic contacts, arcing from slip ring and shaft grounding brushes in rotating machines, PD and corona from the power system which can get coupled to the apparatus under test [1].

In addition to the above sources, other noise sources that can possibly exist in a PD measuring circuit are, ran- 
dom noise from components, harmonics from the mains, periodic pulse currents of thyristor, other pulsive interferences from transformer and interferences from ground connections [2].

In most cases, these external interferences can cause false indications, thereby reducing the credibility of the PD measurements as a diagnostic tool. Ever since this problem was recognized, extensive research work has been pursued in this area and several techniques for suppressing these external interferences have been proposed. Narrow band detectors achieved some success; better results were obtained by balanced bridge arrangements [3] and by pulse discrimination circuits. But, such analog noise suppression methods are cumbersome, require additional equipment, and need critical adjustments, which might not be very easy, under on-site conditions.

With the advent of high-speed computers and fast A/D converters, digital PD measurements became a reality, and soon after many digital methods for noise suppression evolved, such as, moving averages, FFT thresholding, digital filtering (infinite impulse response, IIR and finite impulse response, FIR), adaptive filtering, and more recently, wavelets as well. These methods have yielded varying degrees of success.

In 1988, Feser et al. [4] suggested an FFT-based approach to eliminate DSI; however, this method is computationally intensive and there exists a difficulty in deciding the threshold. In later reports [5,6], they proposed a 128tap FIR filter for filtering DSI, to reduce the processing time and later, also realized a continuously working, multi-stopband, 255-pole FIR filter unit in hardware for suppressing narrow band interferences. In order to reduce the unwanted signals using fixed filtering, their frequency must be known. Hence, this method involves analysis of the signal in frequency domain and requires a filter of very high order, thus, making it also computationally intensive. In 1989, Borsi and Hartje [7] used an adaptive digital filter to suppress DSI. However, such filters are sensitive to noise and cannot track those DSI having very low signal levels. Sher Zaman et al. [8] suggested the application of adaptive signal processing based on principle of decorrelating the PD pulses from the noise, by sampling at a high frequency and inserting a delay in the adaptive system. The advantage of this method happens to be, no prior knowledge of the frequency of noise to be eliminated is required; however, this method involves extensive computation and large processing time. Borsi et al. [9] investigated an adaptive rejection filter, adaptive prediction filter and Kalman filter for suppressing continuous noise and reported their limitations. Su [10] proposed an adaptive filtering method, using terminal calibration pulse as a reference signal. Limitations with the adaptive filtering are, prior knowledge of the frequency of noise and signal characteristics of the reference signal are needed. Also, judicious choice of the parameters is neces- sary for fast convergence. Nagesh and Gururaj [11] compared the performance of various adaptive and non-adaptive digital filters for removing DSI. They reported that, digital filtering method based on a cascaded 2 nd order IIR lattice notch filter was most suitable for this purpose.

Most of the above mentioned methods mainly deal with the removal of sinusoidal interferences and DSI. It is now a commonly accepted fact that, removal of such narrow band interferences is not difficult. However, the main problem that continues to persist is the suppression or removal of pulsive interferences.

Wavelet analysis is a recent development and a powerful tool for detecting transients such as, PD signals, buried in noise and interferences. It has successfully been applied to many areas of science and engineering. Recently, its applicability is also being explored in the area of $\mathrm{HV}$ engineering as well. Massanori et al. [12] reported the application of wavelet analysis in PD measurements, by analyzing the current and sound waves emitted by PD, using Gaussian and Mexican wavelets. They reported that wavelet analysis could be used to diagnose the degradation of insulation. Fujimori et al. [13] analyzed the tree development in solid insulating materials using wavelets. Arli et al. [14] applied wavelet analysis to eliminate sound signals produced due to corona discharge in a void formed by an electrode and a PET film. Yusheng Quan et al. [15] used wavelet analysis on computer simulated PD signals arising due to a cavity, with and without noise. Results showed that the noise could be removed successfully and processing speed was also fast. Florkowski [16] suggested a method for removing noise from PD patterns acquired during on-line PD measurements on a $6 \mathrm{kV}$ motor. In 1997, Wang Hang et al. [17] applied wavelet analysis to extract partial discharge signals from narrow band sinusoidal interferences and reported the effect of various parameters. Shim et al. $[18,19]$ have reported on the possibility of using a wavelet method for denoising PD signals. They examined use of both soft and hard thresholding of wavelet coefficients and highlight its difficulties. Finally, a necessity for exploring more powerful methods is expressed. Thus, from the literature survey, it is evident that, wavelet analysis is emerging as a powerful tool and seems to possess many advantages compared to the existing methods for suppressing interferences from PD signals. Further, it can also be seen that, the application of wavelet-based technique for removal of pulse-shaped interferences, especially, when $P D$ signals and multiple interferences overlap-in-time, has not been examined so far, and hence, forms the subject matter of this paper.

\subsection{PROBLEM DEFINITION}

Of all the external interferences mentioned above, DSI can be identified and eliminated in frequency domain as they have a narrow-band frequency spectrum concentrated around the dominant frequency, whereas, PD pulses 
have a relatively broad-band frequency spectrum. Periodic pulse shaped interferences can be gated-off in time domain to some extent (any PD occurring at the same time instant is lost, and this is undesirable). But, it is very difficult to identify and suppress the stochastic pulse-shaped interferences as they have many characteristics in common (both in time and in frequency) with the PD pulses. Thus, methods to suppress these interferences need to be continually explored in order to improve the sensitivity of on-site and/or on-line PD measurements.

In this paper, the possibility of applying wavelets to suppress the external interference (viz. random, DSI, pulsive and any combination of these present simultaneously and overlapping with PD pulses) is explored, and a method based on multi-resolution signal decomposition (MSD) is proposed. In this regard, both analytical signals and those obtained from actual PD measurements are processed. Results obtained from the proposed wavelet-based method are compared with those obtained from the digital methods proposed earlier $[5,11]$.

\section{WAVELET TRANSFORM 2.1 BRIEF INTRODUCTION TO WAVELET TRANSFORM}

A wavelet is a small wave, which has its energy concentrated in time, and a tool meant for analysis of transients and non-stationary or time varying signals. Just as Fourier analysis consists of decomposing a signal into sine waves of various frequencies, similarly, wavelet analysis is the breaking up of a signal into shifted and scaled version of mother wavelet. Continuous wavelet transform (CWT) of a signal $x(t) \in L^{2}(R)$ is defined as [20]

$$
C W T_{x}^{\psi}(\tau, s)=\frac{1}{\sqrt{|s|}} \int x(t) \psi^{*} \frac{(t-\tau)}{s} d t
$$

where, function $\psi(t)$ is the mother wavelet. It is a prototype for generating the other window functions, which are dilated or compressed and shifted versions of mother wavelet. $\tau$ is the shift operator (translation), $s$ is the scaling function and * stands for complex conjugation. Wavelet transform maps a time-domain signal into a two dimensional array of coefficients, thus localizing the signal in both time and frequency domain simultaneously, whereas, Fourier transform can only give the frequency information. However, CWT is computationally expensive and also generates a lot of redundant data. To circumvent these drawbacks, an effective implementation applicable to discrete signals, called discrete wavelet transform (DWT) was formulated using suitable lowpass and highpass filters, that satisfy certain mathematical constraints. An elegant procedure called the Multi-resolution Signal Decomposition (MSD) technique [20] is implemented through this method, which is the primary reason. for the widespread use of wavelets.

\subsection{DISCRETE WAVELET TRANSFORM (DWT)}

In Discrete wavelet transform, a time-scale representation of a digital signal is obtained using digital filtering technique. Filters of different cutoff frequencies are used to analyze the signal at different scales. The time-domain signal is passed through a series of highpass filters and down-sampled by two to analyze the high frequencies (referred to as detail components), and it is passed through a series of lowpass filters followed by down-sampling by two, to analyze the low frequencies (called approximate components). These highpass and lowpass filters constitute 'quadrature mirror filters', and are exactly half-band filters, thus enabling a perfect error-free reconstruction of the signal. For reconstruction, the above procedure is followed in reverse order i.e. the signals at every level are up-sampled by two and passed through a set of synthesis filters (synthesis filters are derived from analysis filters). For further details, please refer to [20]. Thus, for an $M$ level decomposition-reconstruction, the input signal can perfectly be recovered by adding the reconstructed timedomain approximate component at level $M$ and all the reconstructed time-domain detailed components from level 1 to $M$ i.e.

$$
\begin{aligned}
\text { Reconstructed_signal } & =(\text { Approximate_component })_{M} \\
& +\sum_{j=1}^{M}(\text { Detail_component })_{j} \ldots
\end{aligned}
$$

The MSD method is inherently error-free, and this happens to be a big advantage, since the process by itself does not introduce any additional error.

\section{PROPOSED TECHNIQUE: DE-NOISING BY MSD}

The conventional wavelet denoising method involves calculation of the DWT coefficients for a given signal and then passing the DWT through a threshold (fixed a priori), and followed by reconstruction of the signal by taking the inverse wavelet transform of the modified DWT coefficients. This method is known as soft or hard thresholding. In the present problem, using a threshold does not yield good results, as it is difficult to fix a value of the threshold to be used by a mere inspection of the DWT coefficients. Also, it has been demonstrated in literature $[18,19]$ that, the method based on soft and hard thresholding of DWT suffers from disadvantages and hence, it was not pursued further in this paper.

The proposed technique is basically an off-line technique and has the advantages of low processing time and possibility of better reconstruction, because the suppression of noise/interferences is done in a joint timefrequency domain. This type of denoising consists of two phases. In the first phase, input signal is decomposed into 


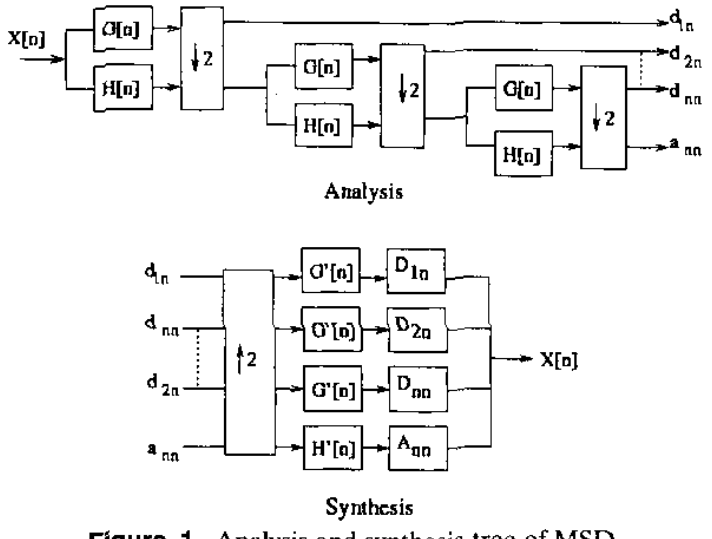

Figure 1. Analysis and synthesis tree of MSD.

approximate and detail components up to a desired number of levels with the help of multiresolution analysis. This is done by first choosing a mother wavelet according to the signal characteristics. Once, the mother wavelet is chosen, decomposition-reconstruction up to the required number of levels is carried out by, scaling and dilating the mother wavelet. The number of decomposition-reconstruction levels can be chosen either by trial and error method or, as in this work, was chosen from the information of sampling frequency. This is because the frequency band of each decomposed component follows a dyadic rule, starting from the Nyquist frequency. Figure 1 illustrates the analysis and synthesis tree explaining the decomposition-reconstruction using multi-resolution signal decomposition. Once the approximate and detail reconstructed time-domain components are computed, the second phase starts. This phase involves identifying those components that correspond to all the interference signals, either by visual inspection or by the knowledge of frequency bands to which the PD pulses belong (this is known from the bandwidth of the PD detector). Finally, the denoised signal is obtained by discarding all the identified components from the summation process. In summary, the proposed wavelet method involves the following three steps:

1. Using a mother wavelet, the input signal is decomposed into a pre-set ( $M$ ) number of levels, and this yields DWT coefficients.

2. The earlier methods worked with these DWTs. But, in the proposed method, the DWTs are reconstructed to yield $M$ levels of time-domain sequences (each of length equal to original signal length). Each of these $M$ levels, correspond to a band of non-overlapping frequencies, and adding all $M$ levels yields the original signal. Thus, a joint time-frequency representation of the input signal is obtained.

3. Denoising in the present method essentially consists of a visual inspection of the $M$ reconstructed time-domain components (or levels), and identifying those that lie within the pass-band of the PD band-pass filter used. In some special cases, one or two extra bands on either side of the band-pass may have to be chosen. Depending on sampling frequency and levels of decomposition, it is easy to locate the bands that are to be retained. The rest of the time-domain components are unwanted and discarded from the summation process.

As, the algorithm for recovering the denoised signal is relatively fast, once the interfering frequency bands (or levels) are identified, the wavelet-based approach has a greater possibility of being implemented on-line.

\subsection{ISSUES IN WAVELET METHOD}

During the implementation of the MSD approach, chice of some parameters connected with MSD method has to be made. This is inherent to the method, and concerns issues like choice of mother wavelet, number of levels of decomposition, etc. They are briefly addressed below:

1. Selection of mother wavelet. This is a core question often asked, but a generalized answer seems to be still elusive even to signal processing experts. Also, no direct answers are available in literature. With this being reality, its choice in the present work was based on a trial-anderror and guided by hints published in literature on similar type of work. Being well aware of this issue, the authors have examined many wavelets and found Daubechies' wavelet most suitable. Further, many practical examples have been included to demonstrate that the wavelet selected was good enough for the task. A detailed comparative study of all wavelets was not exclusively reported, as this was not within the identified scope of the paper.

2. Selection of number of levels for decompositionreconstruction. The goal was to have sufficient resolution in frequency bands at lower frequencies, as low frequency pulsive interferences were also to be eliminated. If lesser levels were used, then PD signals and the low frequency interferences would be clubbed together, and hence difficult to segregate. Keeping this in mind, ten decomposition-reconstruction levels were chosen and found to be sufficient in most of the cases.

3. Applicability to practical applications. Once a mother wavelet and number of levels are decided for a given test setup and ambient/site conditions, the remaining procedure is more or less simple to implement. However, if any change in measuring circuit or test environment occurs, then an operator intervention is essential to re-examine and identify which of the reconstruction components are to be retained. This strategy has worked well, at least, in the examined lab test setup and prevalent ambient conditions. Also, simplicity of the method makes it not too dif- 
ficult to re-adjust, when a new ambient condition comes into existence.

In summary, it is true that the choice of mother wavelet, number of levels, practical applications, etc. are a few signal processing issues of this approach, and should not be looked upon as a deterrent for its use. But, it must be pointed out here that, the foremost objective of this paper was to find a method capable of effectively suppressing all types of interferences, even when PD signals and interferences overlap-in-time. On this count, original and novel solutions have been reported.

\section{DIGITAL SIMULATION SETUP}

\subsection{NEED FOR SYNTHESIS OF INPUT SIGNAL}

For studying the performance of different noise suppression techniques, it is necessary to consider the influence of all the parameters of each method on the final result. These influences can critically be evaluated, if the input signal, noise/interferences, source and output signals are all exactly known. In addition, the relevant parameters must easily be controllable. Hence, the input signal and interferences need to be synthesized in a realistic manner. This approach also facilitates the reproducibility of the results by other researchers. Although, practical experimental setup is another source of such signals, the feasibility of controlling individual components and their relevant parameters is often time consuming and clifficult, if not impossible. Hence, a digital generation of signals was initially adopted to fine-tune the methods, and later on, they were verified with the actual signals.

\subsection{CHOICE OF SIMULATION PARAMETERS}

A realistic simulation of PD pulses corrupted with noise/interferences was carried out by considering most of the factors typically encountered during on-site or online PD measurements. The input signal was synthesized by superposing PD pulses of known shape with DSI and random noise. The parameters chosen for simulation are discussed below:

- PD Pulses. PD pulses were simulated by an exponentially decaying function, as was done by other researchers in the past [11]. These pulses were convolved with the impulse response of a 2 nd order Butterworth filter ( $\mathrm{fl} / \mathrm{f} 2: 30$ $\mathrm{kHz}-300 \mathrm{kHz}$ ) to generate the signal corresponding to the quasi-integrated output of the PD detector. In order to take the pulse resolution time into account, a train of pulses were analyzed. Oscillatory pulse and pulses of different amplitudes were also analyzed, to give a better picture of the performance of various denoising techniques considered.

- Background noise. In order to simulate the thermal noise generated by the detection system itself and the
Table 1. Characteristics of DSI used in simulation.

\begin{tabular}{lllllllll}
\hline f $(\mathrm{kHz})$ & 200 & 300 & 450 & 500 & 620 & 700 & 800 & 980 \\
$\mathrm{INR}^{*}(\mathrm{~dB})$ & 1.8 & 10.2 & -0.9 & 2.9 & 10.8 & -6.4 & 2.7 & -6.4
\end{tabular}

*Interference to noise ratio.

background noise, white noise of zero mean and of varying power was added to get different SNR.

- DSI. The DSI(s) were generated as amplitude modulated sine-waves with $40 \%$ modulation, and a constant modulating frequency of $1 \mathrm{kHz}$. Table 1 describes the characteristics of the eight DSI used in the simulation.

- Pulsive interferences. These are considered only in case of actual PD signals.

\subsection{BASIS FOR COMPARISON OF TECHNIQUES}

During the process of denoising, in addition to removal of noise components, the signal component whose frequency is very close to the interfering frequency ranges also gets removed (although, to a lesser extent). Thus, the signal component to be recovered suffers from both attenuation and distortion. These two features may be quantified by defining the peak amplitude and the correlation coefficient. An acceptable noise suppression method should reject or suppress all the interferences and noise with minimum attenuation and distortion of the PD pulse. In order to compare the performance of various methods, the following indices have been considered:

\subsubsection{SIGNAL TO NOISE RATIO}

The signal to noise ratio (SNR), illustrates the effectiveness of denoising operation [21]. It is defined as

$$
\mathrm{SNR}=10 * \log \frac{\sum_{i=1}^{N} Y^{2}(i)}{\sum_{i=1}^{N}(X(i)-Y(i))^{2}}
$$

where, $X(i)$ is the original reference signal, $Y(i)$ is the denoised signal and $N$ is the number of samples. A positive value of SNR implies a greater power of the signal as compared to the noise and a negative value of SNR implies a greater power of noise as compared to the signal.

As in practical records, there is no such reference signal $X(i)$ to compute SNR, only the extent of noise suppressed can be estimated. The normalized noise reduction was computed as:

reduction in noise level $(d B)=10 * \log \sum_{i=1}^{N} \frac{1}{N}(Z(i)-Y(i))^{2}$ 
where, $Z(i)$ is the noisy signal acquired, $Y(i)$ is the denoised signal and $N$ is the number of samples.

\subsubsection{REDUCTION IN PD PULSE AMPLITUDE}

The percentage reduction in PD pulse amplitude of the denoised signal with respect to the reference signal is defined as

$$
\text { \%reduction }=\frac{X-Y}{X} * 100
$$

where, $X$ is the peak amplitude (positive going peak in this case) of the original (reference) PD pulse and ' $Y$ ' is the peak amplitude of the denoised pulse recovered. In this simulation, since a pulse sequence is used, so the percentage reduction in the peak amplitude of the pulse recovered after denoising with respect to the corresponding pulse in the input sequence is calculated.

\subsubsection{CROSS CORRELATION COEFFICIENT}

In order to compare the extent of distortion the PD signal undergoes due to different denoising methods, the correlation coefficient between input and denoised signal was computed [21]. It is defined as

$$
R x y(m)=\sum_{i=0}^{M-|m|-1} X(i) Y(i+m)
$$

where, $X(i)$ is the input signal $(1 \ldots N), Y(i)$ is the denoised signal $(1 \ldots N)$, and $R x y(m)$ is the correlation coefficient ranging from $-(N-1)$ to $(N+1) . R x y(m)$ indicates the degree of similarity and hence the extent of distortion as well, in terms of the pulse shape of the denoised and the original signal.

In general, a denoising method is considered acceptable, when SNR is high, the percentage reduction in amplitude is low, and the distortion in pulse shape is minimum. It must be mentioned that, while calculating the SNR and correlation coefficient, the edge effects and the phase shift in the denoising method was taken into account.

\section{RESULTS AND DISCUSSION}

The denoising methods under consideration shall initially be compared with mathematically generated signals, followed by their ability to denoise practical PD signals.

\subsection{PROCESSING OF SIMULATED SIGNALS}

Three PD pulse sequences were simulated using the procedure explained in Section 4. These sequences called Signal-1, Signal-2 and Signal-3, comprise the following components:

Signal-1: PD pulse train of equal amplitude + DSI + random noise.
Table 2. Denoising techniques.

\begin{tabular}{cl}
\hline Method & \multicolumn{1}{c}{ Technique } \\
\hline A & IIR filtering \\
B & 128 tap FIR filtering \\
C & MSD (wavelet) \\
\hline
\end{tabular}

Signal-2: PD pulse train of different amplitudes + DSI

+ random noise.

Signal-3: A single PD oscillatory pulse + DSI

+ random noise.

Signal-1 is a sequence of 40 PD pulses of amplitude 3.7 units, where each PD pulse is separated by $10 \mu$ s and to it is added random noise and DSI of very high amplitude. Signal-2 is more or less similar to Signal-1, except for varying PD pulse amplitudes. Signal-3 contains a single oscillatory PD pulse of peak amplitude about 0.3 units and duration $60 \mu s$, and is mixed with random noise and interference of high amplitude. For this signal, a bandwidth of $f_{1} / f_{2}=100 \mathrm{kHz} / 300 \mathrm{kHz}$ was used. The sampling frequency was $5 \mathrm{MHz}$.

Each of these signals has been analyzed for three different noise levels (SNR), wherein the power of DSI as well as the random noise was progressively increased. Three SNR values are considered and designated as SNR1, SNR-2 and SNR-3 corresponding to $-21.59 \mathrm{~dB},-36.79$ $\mathrm{dB}$ and $-49.66 \mathrm{~dB}$ respectively. These sequences were
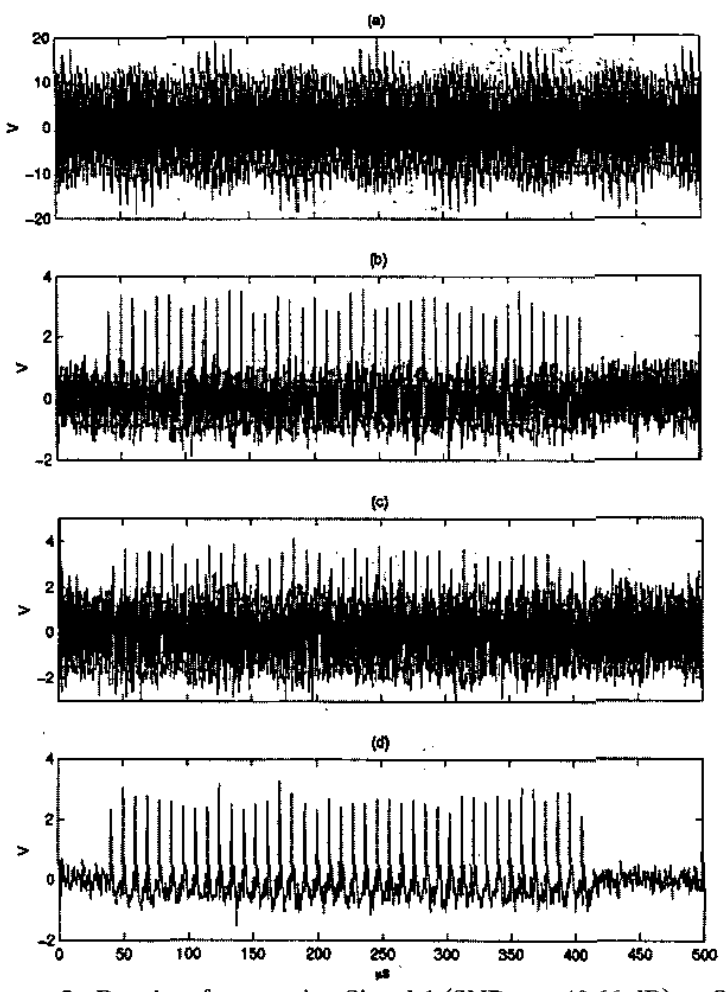

Figure 2. Results of processing Signal-1 ( $\mathrm{SNR}=-49.66 \mathrm{~dB})$. a, Signal-1; b, Method A; c, Method B; d, Method C. 
processed by the three denoising methods (all programs were implemented in MATLAB) listed in Table 2.

All the three methods yield good results, when the signals processed have high signal to noise ratios (SNR-1). But, as the SNR of the input signal decreases, namely, in case of SNR-2 and SNR-3, the denoised signal obtained by methods $\mathrm{A}$ and $\mathrm{B}$ progressively suffers higher distortion (indicated by a low correlation coefficient), as well as, a decrease in amplitude of the recovered PD pulse. On the contrary, method $C$ yields comparatively far better results for all SNR levels investigated (for all cases of Signal-1, Signal-2 and Signal-3). Due to brevity of space, only the worst-case condition i.e. SNR-3 for the three signal cases are discussed.

In case of Signal-1, as seen from Figure 2a, peak amplitude of the noisy signal is about 20 units and SNR. of the corrupted signal is $-49.66 \mathrm{~dB}$. The PD pulses are completely buried in noise and it is impossible to identify their presence by visual inspection. Processing of this signal by method A yields the signal shown in Figure $2 b$, which has an SNR of $5.22 \mathrm{~dB}$, thus resulting in a net SNR improvement of about $55 \mathrm{~dB}$. Whereas, processing by method B yields signal shown in Figure $2 \mathrm{c}$ having SNR of $1.68 \mathrm{~dB}$ thereby resulting in a net improvement of $51.34 \mathrm{~dB}$. Although the improvement in SNR by these two methods appears to be comparable and satisfactory, the correlation coefficients for both the methods are low ( 0.66 and 0.39 , respectively), as seen from Table 3 . This implies that, there occurs a heavy distortion in the PD pulse shape. Also, it must be mentioned here that, filtering the interfering frequency components by these two methods was cumbersome and sensitive process. That is, the signal had to be first transformed into the frequency domain and then the desired frequency response (namely, the stop-bands) of the filters had to be selected. Even a slightly different choice of the frequency characteristics of the stop-bands resulted in a completely different output; thus, it called for a very careful selection of the stop-bands. One example to illustrate this aspect will be considered when results of practical PD signals are considered in the next section.

Processing Signal- 1 by method $C$ results in the signal shown in Figure $2 \mathrm{~d}$ having an SNR of $15.62 \mathrm{~dB}$. The overall improvement in SNR in this case is about $65 \mathrm{~dB}$. Also, the correlation coefficient for the denoised signal is 0.89 (Table 3), demonstrating a successful recovery of the PD pulse shape as well. The output obtained by this method

Table 3. Results of denoising Signal-1.

\begin{tabular}{|c|c|c|c|c|}
\hline Method & $\begin{array}{c}\text { SNR of } \\
\text { processed } \\
\text { Signal (dB) }\end{array}$ & $\begin{array}{l}\text { Correlation } \\
\text { Coefficient }\end{array}$ & $\begin{array}{c}\% \\
\text { Reduction } \\
\text { in amplitude }\end{array}$ & $\begin{array}{c}\text { Improvement } \\
\text { in } \\
\text { SNR. (dB) }\end{array}$ \\
\hline A & 5.22 & 0.66 & 21.6 & 54.88 \\
\hline B & 1.68 & 0.39 & 16.2 & 51.34 \\
\hline C & 15.62 & 0.89 & 22.3 & 65.28 \\
\hline
\end{tabular}

Table 4. Results of denoising Signal-2.

\begin{tabular}{|c|c|c|c|c|}
\hline Method & $\begin{array}{c}\text { SNR of } \\
\text { processed } \\
\text { Signal (dB) }\end{array}$ & $\begin{array}{l}\text { Correlation } \\
\text { Coefficient }\end{array}$ & $\begin{array}{c}\% \\
\text { Reduction } \\
\text { in amplitude }\end{array}$ & $\begin{array}{c}\text { Improvement } \\
\text { in } \\
\text { SNR }(\mathrm{dB})\end{array}$ \\
\hline $\mathrm{A}$ & 6.12 & 0.47 & - & 55.78 \\
\hline B & 0.79 & 0.26 & - & 50.45 \\
\hline $\mathrm{C}$ & 16.45 & 0.89 & 18.9 & 66.11 \\
\hline
\end{tabular}

is definitely far superior compared to the other two methods. Further, processing is relatively easier, as denoising is done in the time-frequency domain, and it is convenient to identify the disturbing frequency ranges. For processing of Signal-1 (at all SNR levels) "Daubechies 30" was chosen as mother wavelet and the decomposition was done up to 10 levels. The denoised signal was obtained by choosing only the reconstructed approximate component at level 4. Processing was also done with other siblings of "Daubechies" and "Symlets" as mother wavelets, but the denoised signal did not show any great improvement. Studies with other mother wavelets were also conducted. However, experience showed that better results were obtained with "Daubechies 30".

In order to analyze the performance of the three methods to process PD pulses of varying amplitudes (which is usually the case), Signal-2 was next considered. The results are summarized in Table 4 and Figures $3 b, 3 c$ and $3 d$
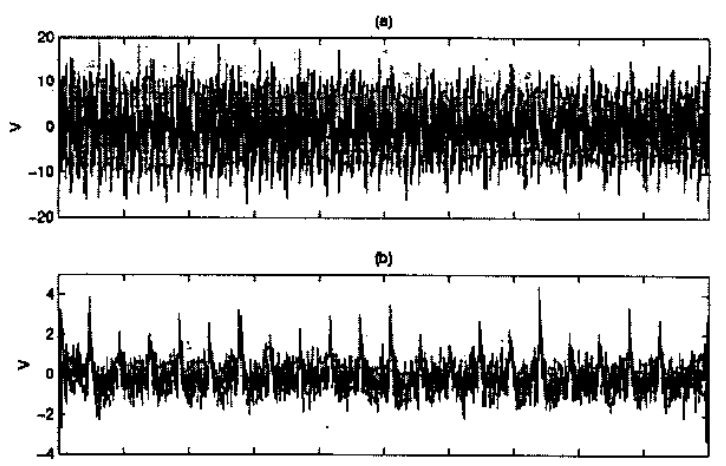

(c)
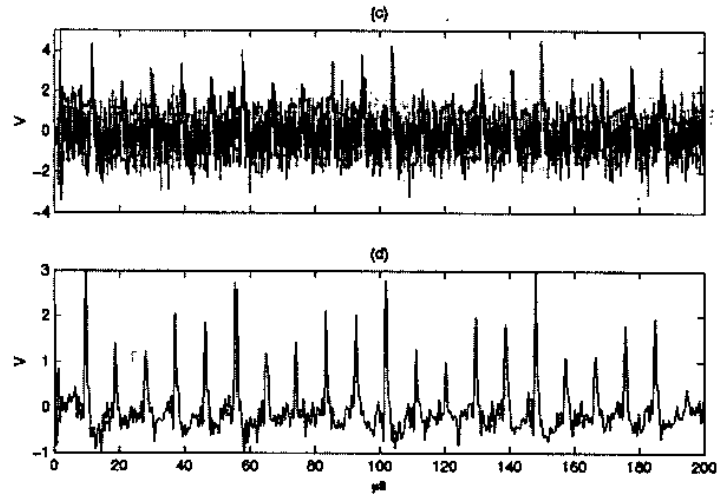

Figure 3. Results of processing Signal-2 (SNR $=-49.66 \mathrm{~dB}$ ). a, Signal-2; b, Method A; c, Method B; d, Method C. 

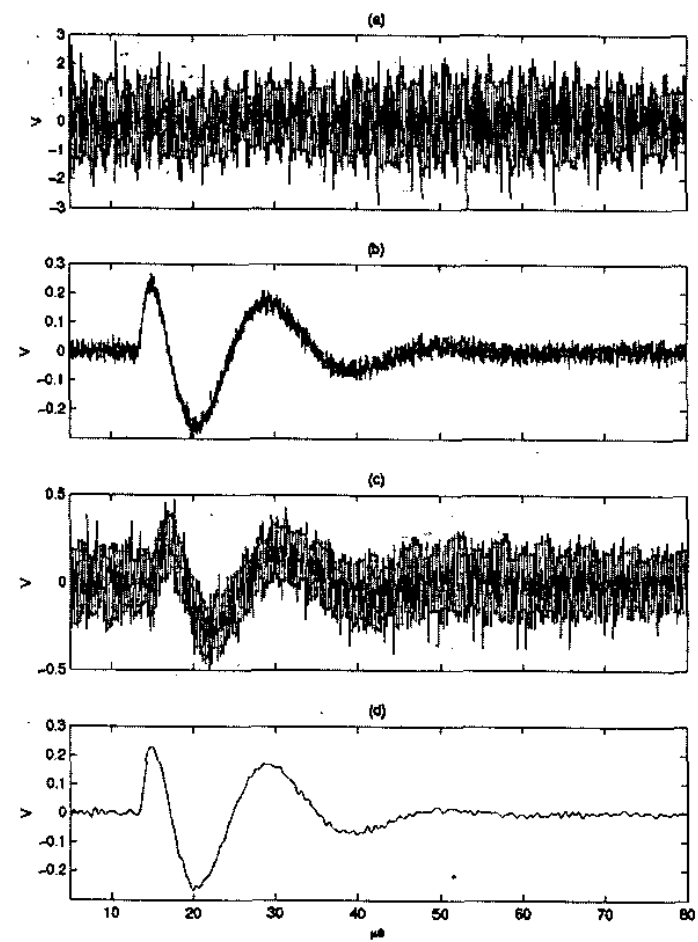

Figure 4. Results of processing Signal-3 (SNR $=-49.66 \mathrm{~dB}$ ). a, Signal-3; b, Method A; c, Method B; d, Method C.

depict the denoised signals due to the three methods respectively. As can be seen, the denoised signals show a trend similar to what was observed in case of Signal-1.

From the results discussed so far and the estimated correlation coefficients, it can be summarized that the PD pulses recovered are distorted to some extent. To further analyze this aspect in detail, Signal-3 consisting of a single oscillatory PD pulse of comparatively longer duration and mixed with DSI and noise was considered next. The results of processing this signal by method $A$ and $B$ are shown in Figures $4 \mathrm{~b}$ and $4 \mathrm{c}$, respectively. The correlation coefficients of 0.86 and 0.49 clearly imply the extent of distortion in pulse shape. Whereas, method $\mathrm{C}$ was able to completely recover the original oscillatory pulse (Figure 4d). The correlation coefficient here was 0.99 , thus implying negligible distortion of pulse shape. For this example, denoising by method $\mathrm{C}$ was done by decomposing the signal up to 10 levels and using "Daubechies 7" as the mother wavelet. The denoised signal was reconstructed by choosing only the approximate component at level 4 . Results for this case are summarized in Table 5.

From the simulation results discussed above, it emerges that, processing by method $\mathrm{C}$ resulted in the least distortion of PD pulse shape (as indicated by high correlation coefficient for all cases) and also a significant improvement in SNR, compared to the other two methods A and B. This is because, in method $\mathrm{C}$, the signal is being ana-
Table 5. Results of denoising Signal-3.

\begin{tabular}{clll}
\hline & $\begin{array}{l}\text { SNR of } \\
\text { processed } \\
\text { Method }\end{array}$ & $\begin{array}{l}\text { Correlation } \\
\text { Signal (dB) }\end{array}$ & $\begin{array}{l}\text { Improvement } \\
\text { in }\end{array}$ \\
\hline A & 30.65 & 0.86 & 80.31 \\
B & 2.80 & 0.49 & 52.46 \\
C & 50.2 & 0.99 & 99.86 \\
\hline
\end{tabular}

lyzed at different frequency levels with different time resolutions, thereby making it comparatively easier to identify and reject the interfering frequencies.

\subsection{PROCESSING OF PRACTICAL RECORDS}

It is not always convenient to generate all types of noise/interference by digital simulation, especially pulsive interferences. Also, simulation of propagation characteristics of the PD pulses in power apparatus and their influence on its shape are complex. Therefore, to assess the performance of the different noise suppression methods, practical PD signals were generated from carefully planned experiments. During this exercise no specific effort was made to suppress ambient noise and interferences, so that PD signals corrupted by noise/interferences could be acquired.

\subsubsection{EXPERIMENTAL SETUP AND DATA ACQUISITION}

Figure 5 shows the schematic diagram of the experimental setup. A point-plane gap configuration was chosen as the source of PD. The bandwidth of the PD detector chosen is usually a compromise between PD pulse resolution time and error due to quasi-integration $[2,22]$. A constant bandwidth of $30 \mathrm{kHz}-300 \mathrm{kHz}$ was used and PD signals were detected with a reasonably high sensitivity.

The amplified and quasi-integrated PD signal available at the PD detector output (without the sine-wave) was acquired using a 10-bit digitizer (Sony-Tektronix, RTD 710A). The digital data was transferred via GPIB to a PC using the 'Direct I/O option' in the HP-VEE programming environment. The sampling rate was chosen such that

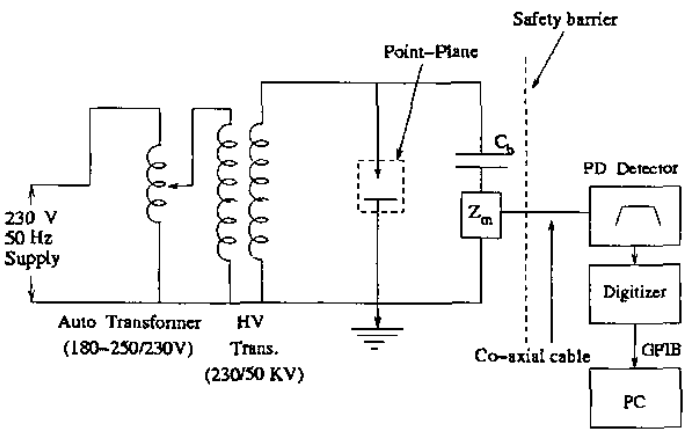

Figure 5. Schematic of experimental setup. 
the recorded signal corresponds to one power frequency cycle. So a sampling rate of $100 \mathrm{~ns}$ was used, resulting in a record length of $256 \mathrm{k}$ samples. All data acquisitions were carried out ensuring a maximum possible full-scale deflection on the digitizer. Many signals were acquired, from which a set of four signals (Set-1 to 4) with different noise/interferences and PD source conditions are presented here. The selected four examples represent some of the most difficult cases to denoise and hence were intentionally included here. It should be noted that, the signal acquired does not have the correct phase information, since the quasi-integrated PD detector output does not contain the $50 \mathrm{~Hz}$ component. This is not a shortcoming. However, correct phase information can be obtained by using a suitable external trigger (with appropriate circuit modification) during data acquisition.

\subsubsection{PROCESSING OF PRACTICAL PD SIGNALS}

The practical PD signals were processed with noise suppression methods A, B and C (see Table 2). The salient features of data processing and results obtained for each of the four signals are discussed below:

Set-1: This signal was acquired by feeding a stray pickup from a wire connected to the input amplifier along with the calibration pulse of the PD detector. In this acquisition, power supply to the circuit was connected, but not energized. Figure 6a shows the signal acquired, and from the plot it can be seen that, the two calibration pulses (about $10 \mathrm{~ms}$ apart) are visible, but the noise level is also comparable to amplitude of the calibration pulse. At the time of experimentation, it was found that this noise was significantly reduced by turning off the electronic power supplies, SMPS and monitors of the personal computers within the vicinity of the test circuit. Inspection of the acquired signal showed that the noise was periodic, nonsinusoidal, and had a fundamental frequency of about 100 $\mathrm{kHz}$ (which lies within the pass band of the PD detector).

For purpose of clarity, Figure $6 \mathrm{~b}$ shows a portion of the signal chosen for processing. A careful inspection of Figure $6 \mathrm{~b}$ reveals that, the $\mathrm{PD}$ calibration pulse main frequency is very close to the main frequency of the interference, due to which the two signals have almost merged with each other. Processing this portion of the signal by method A yields the signal shown in Figure $6 \mathrm{c}$. The quantum of noise reduction is about $-19.3 \mathrm{~dB}$. The denoised signal obtained from Method B is shown in Figure 6d, wherein about $-14.2 \mathrm{~dB}$ reduction in noise level was achieved. As, in practical records, there is no reference signal to compute SNR (as per equation 3), only the extent of noise suppressed can be estimated. This noise reduction is computed as ten times the logarithm of the square of the normalized difference between the original and denoised signal (equation 4). From these plots it can be seen that, although the filtering methods $\mathrm{A}$ and $\mathrm{B}$ are
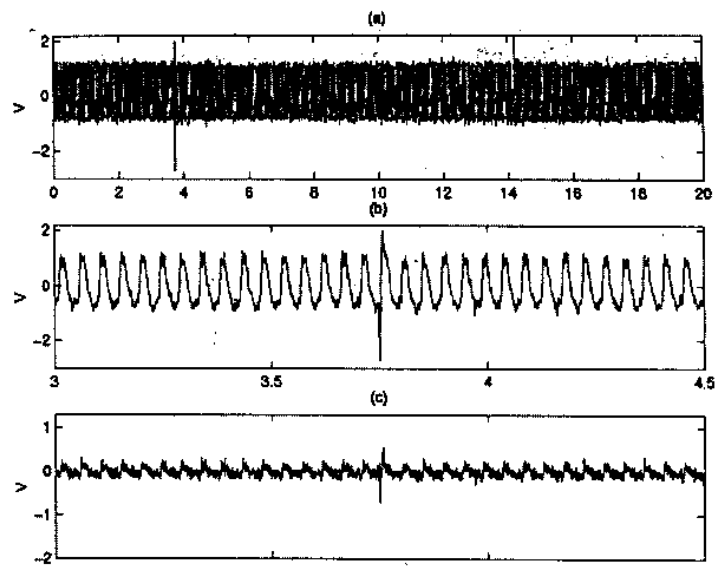

(d)
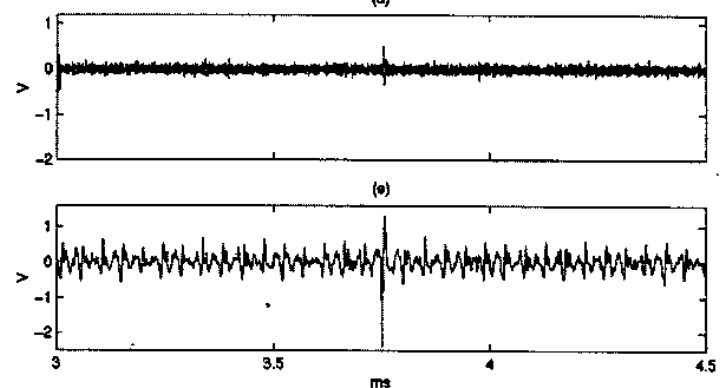

Figure 6. Results of processing practical signal Set-1. a, Acquired signal; b, Portion of (a) processed; c, Method A; d, Method B; e, Method C.

able to reject noise, they have also distorted the PD pulse shape (as seen from zoomed view in Figure 7). Also, in order to denoise the signal by these methods, some difficulty was encountered in selecting the optimal frequency response characteristics of the filters. Even a small change in the frequency response of these filters, resulted in either a very high attenuation of the PD pulses (as can be seen from Figure 8) or no significant reduction in the noise level. Figures $8 \mathrm{~b}$ and $8 \mathrm{c}$ are the outputs obtained by meth-

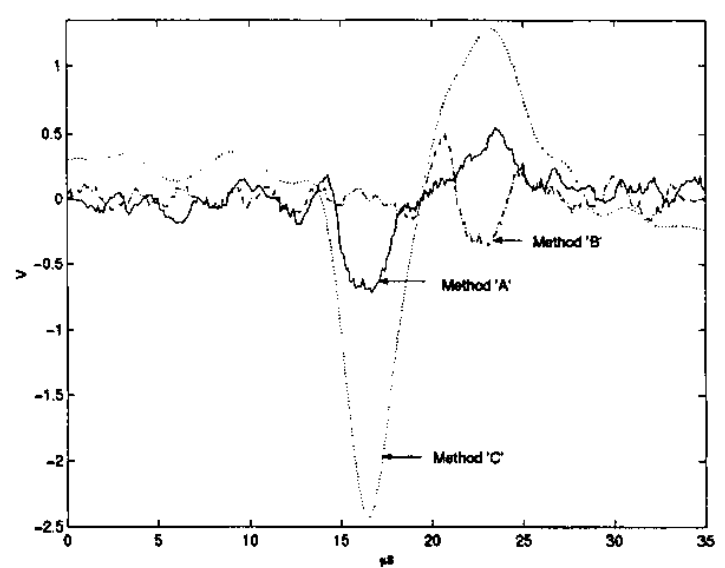

Figure 7. Zoomed view of denoised PD pulse (Set-1) obtained by methods $\mathrm{A}, \mathrm{B}$ and $\mathrm{C}$. 

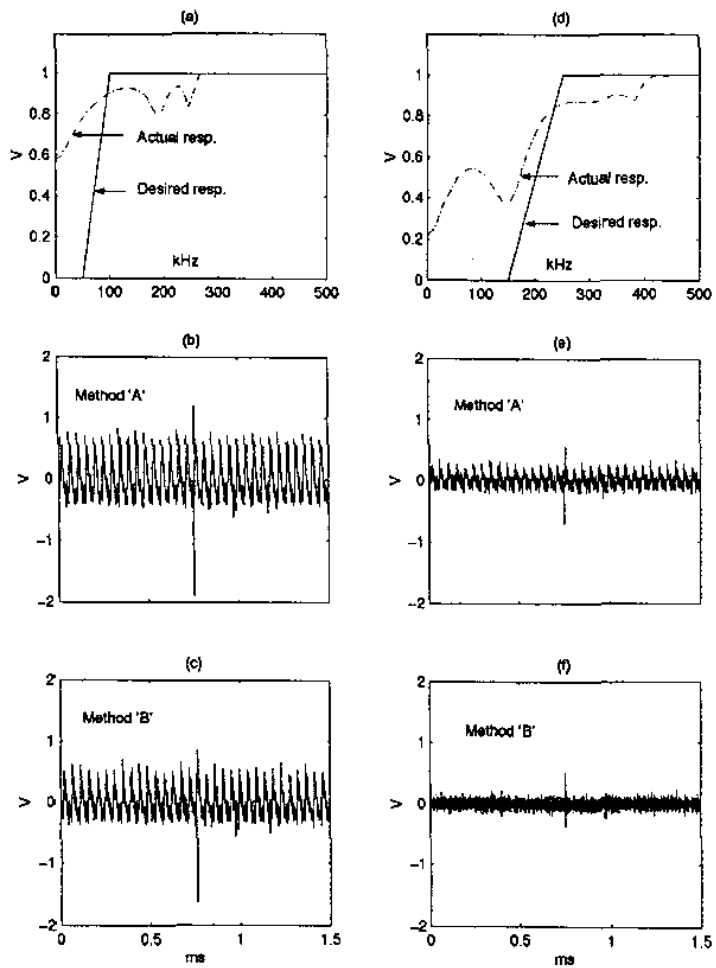

Figure 8. Results of processing signal in Figure $6 \mathrm{~b}$ by methods $\mathrm{A}$ and $\mathrm{B}$ for changed filter characteristics. a, Frequency characteristics; b, Output of method A for frequency characteristics in (a); c, Output of method B for frequency characteristics in (a); $\mathrm{d}$, Changed frequency characteristics; e, Output of method A for frequency characteristics in (d); f, Output of method B for frequency characteristics in (d).

ods A and B respectively for the filter response as shown in Figure 8a. Similarly, Figures $8 \mathrm{e}$ and $8 \mathrm{f}$ are the responses obtained by methods $\mathrm{A}$ and $\mathrm{B}$ respectively, for a small change in the frequency response shown in Figure $8 d$. From the plots it is seen that a slight shift in the frequency characteristics of the filters results in a significant attenuation of the PD pulses.

For processing of this signal by method $\mathrm{C}$, the signal was decomposed up to 10 levels using "Daubechies 15 " as mother wavelet. Figure 9 shows the reconstructed detailed components $\left(D_{1}\right.$ to $\left.D_{10}\right)$ up to level 10 . The denoised signal was obtained by summing the reconstructed detail components at levels 5,6 and 7 only. The rest were discarded. It is seen that, about $-8.8 \mathrm{~dB}$ noise reduction has been obtained, which is the highest compared to the other two methods. From the denoised signal, in Figure $6 e$, it is seen that, a significant amount of low frequency noise has been removed, when compared to the results obtained from methods $\mathrm{A}$ and $\mathrm{B}$. This example illustrates that the multiresolution feature helps in obtaining a fine frequency separation, while the other methods were unsuccessful. Additionally, there is also a better recovery of pulse shape and pulse amplitude reduction is far lesser,

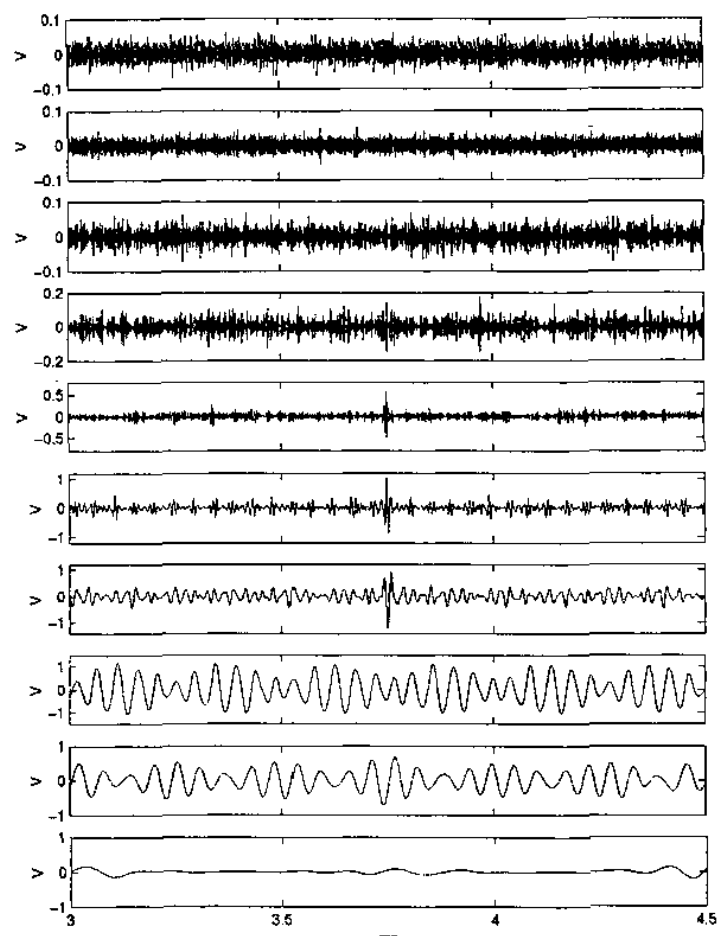

Figure 9. Reconstructed detail components $\left(D_{1}\right.$ to $\left.D_{10}\right)$ up to 10 levels for signal (Set-1) shown in Figure $6 \mathrm{~b}$.

namely, about $20 \%$ whereas, the pulse amplitude reduction in methods $\mathrm{A}$ and $\mathrm{B}$ was $73 \%$ and $80 \%$ respectively (as can be seen in Figure 6).

Set-2: This signal is similar to Set-1, and mainly contains a calibration pulse together with the noise and interferences present in the lab and picked up by the wire connected to the input amplifier. In this case, the interference is more sinusoidal compared to the previous case. Figure $10 \mathrm{a}$ is the recorded signal and Figure 10b shows the portion of the signal chosen to be processed. From Figure 10b, it can be seen that the frequency of the interference is in fact very close to the main frequency of the PD pulse, representing an onerous task for any denoising method.

Figures $10 \mathrm{c}, 10 \mathrm{~d}$, and $10 \mathrm{e}$, show the denoised signals obtained by methods $\mathrm{A}, \mathrm{B}$ and $\mathrm{C}$, respectively. From the denoised signals, it is clear that, processing by method $\mathrm{A}$ does not effectively remove the continuous periodic interference, whose frequency is very close to the frequency of the PD pulse. Further, there is a higher attenuation of the PD pulse, which makes it very difficult to identify it in the denoised signal. Denoising by method B produced slightly better results compared to method $\mathrm{A}(-12.5 \mathrm{~dB})$ and yielded about $-1.8 \mathrm{~dB}$ reduction in noise level, thus resulting in a considerable reduction of the interfering frequencies. But, it is accompanied by about $84 \%$ reduction in pulse amplitude (peak to peak) as well, implying a 

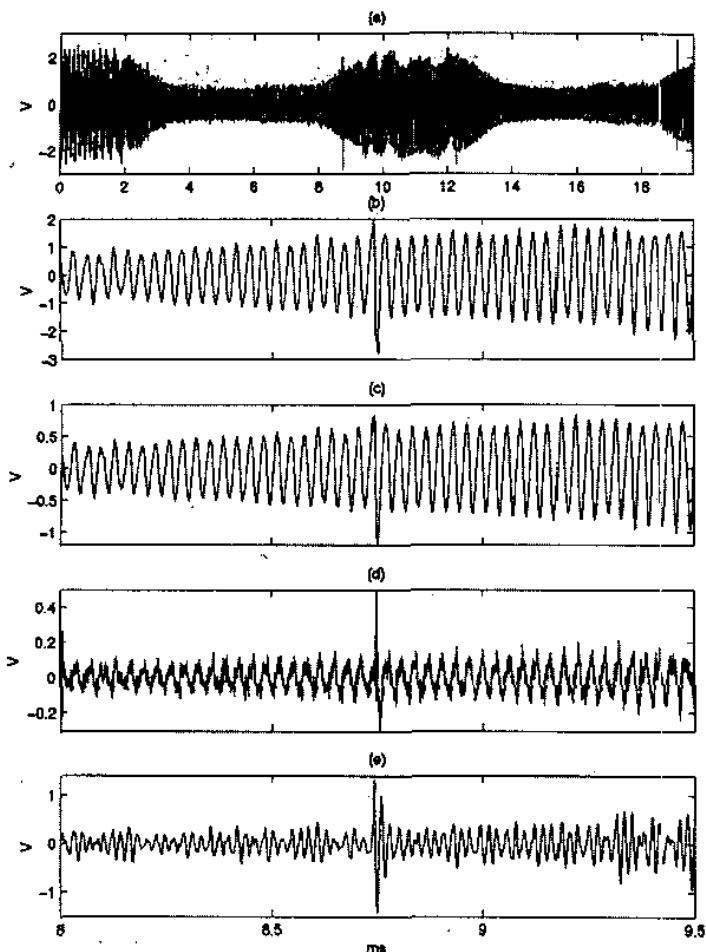

Figure 10. Results of processing practical signal Set-2. a, Acquired signal; b, Portion of (a) processed; c, Method A; d, Method B; e, Method C.

heavy attenuation of PD pulse amplitude. As in the previous case, here also, there existed a difficulty in the choice of the desired frequency response characteristics of the filters, as the interfering frequency was very close to the frequency to be recovered. Also, the filter orders had to be increased (IIR-34, FIR-230), to achieve very narrow stop bands required in this particular case.

In order to process this signal by method $\mathrm{C}$, "Daubechies 12" was used as the mother wavelet and the signal was decomposed up to 10 levels. The Figure 11 shows the decomposition components $\left(D_{1}\right.$ to $\left.D_{10}\right)$ up to 10 levels. After inspection of all the components in Figure 11 , the denoised signal was obtained by adding reconstructed detail components at levels 5,6 and 7 only. This method resulted in about $-1.2 \mathrm{~dB}$ improvement in noise level and a $33.3 \%$ reduction in pulse amplitude (peak to peak), which is significantly better than the results produced by the other two methods, as can be seen from Figure 10e. The signal artifacts seen around $9.3 \mathrm{~ms}$ and 9.5 $\mathrm{ms}$ in Figure 10e will not classified as PD. Lastly, this method recovers the PD pulse with minimum distortion in pulse shape and introduces a lesser reduction in pulse amplitude.

Set-3: This signal contains corona pulses in the time interval 1-3 ms (obtained from a point-plane configuration) and shown in Figure 12a. Additionally, two high ampli-

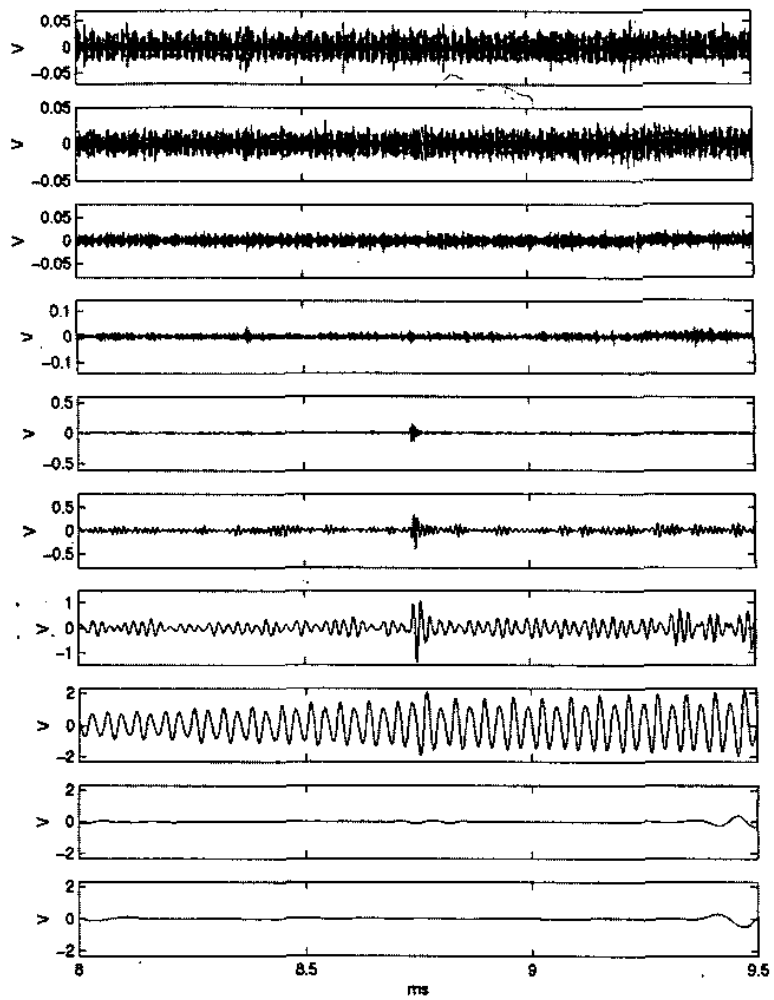

Figure 11. Reconstructed detail components $\left(D_{1}\right.$ to $\left.D_{10}\right)$ up to 10 levels for signal (Set-2) shown in Figure 10b.

tude, low frequency, phase-stable, pulsive interference, separated by about $10 \mathrm{~ms}$ are also observed in the recorded signal. Further, a closer inspection (of Figure 12b) reveals that, the corona pulses are also superimposed on another low frequency pulse-shaped interference of comparable magnitude, which has to be suppressed as well. Figures $12 \mathrm{~b}, 12 \mathrm{c}, 12 \mathrm{~d}$ and $12 \mathrm{e}$, show the portion of the signal processed, and the denoised signals obtained by methods $\mathrm{A}, \mathrm{B}$ and $\mathrm{C}$, respectively. Of course, time-gating can be used to remove the pulse shaped interference superimposed on the corona pulses, but, in this particular case, it will result in the removal of a significant number of the corona pulses as well. This is not desirable. The goal is to suppress the interference, while at the same time recover all the PD pulses as well.

From the figures, it is seen that denoising by method $\mathrm{A}$ and $B$ could not suppress the pulsive interferences effectively. Though there is an appreciable reduction in the amplitude of the pulsive interference $(65 \%$ and $76 \%$, respectively), the amplitude of corona pulses have also been reduced (by $42.8 \%$ and $57 \%$, respectively), which is undesirable.

From Figure $12 \mathrm{e}$, it can be seen that method $\mathrm{C}$ completely removes the two pulsive interferences and extracts the corona pulses with negligible reduction in their amplitude and shape. Further, the interference superposed on 

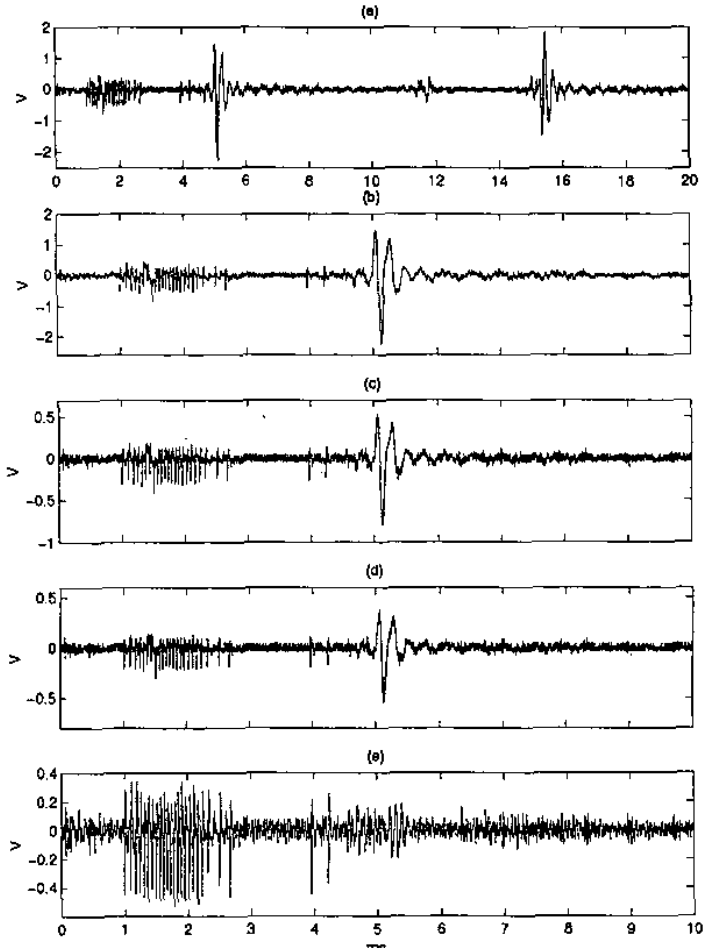

Figure 12. Results of processing practical signal Set-3. a, Acquired signal; b, Portion of (a) processed; c, Method A; d, Method B; e, Method C.

the corona pulses has also been successfully removed, without distorting the PD pulses. For processing by this method, the signal was decomposed up to 10 levels (Figure 13 shows $D_{1}$ to $D_{10}$ detailed components) by using "Daubechies 16 " as the mother wavelet. The denoised signal was obtained by adding reconstructed detail components at levels $4,5,6,7,8$ and 9 only.

From the denoised signal plots, it is seen that, the pulsive interference that overlaps in time with the corona pulses has been completely removed, only when the signal was treated with method $\mathrm{C}$ (Figure 12e). Further, the corona pulses that have been recovered have not suffered any distortion. At the same time, the other two methods were not so successful in this count. Thus, this example demonstrates the effectiveness of the wavelet-based method in removing noises even from such critical cases, namely, when PD pulses and pulsive interferences signals overlap in time. Such explicit examples have, to the best knowledge of the authors, not been reported and discussed earlier. Thus, it demonstrates the novelty of the method.

Set-4: This signal was recorded under the presence of corona produced by the point-plane gap. In addition to the existing background noise, a sinusoidal interference of $612 \mathrm{kHz}$ was externally injected to the input of the PD detector using a signal generator to simulate an AM radio station.

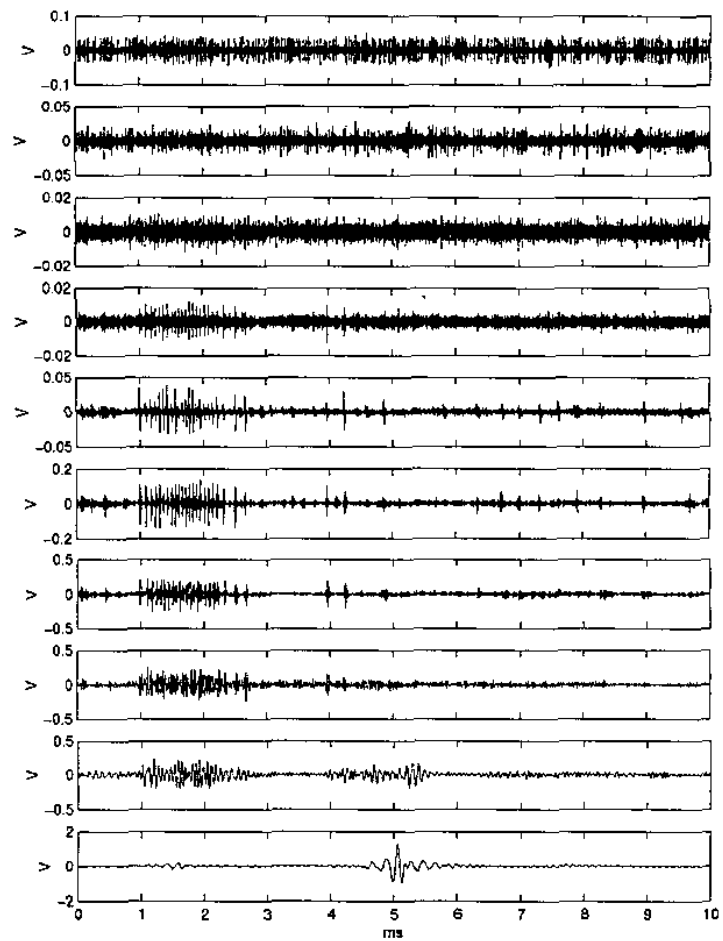

Figure 13. Reconstructed detail components $\left(D_{1}\right.$ to $\left.D_{10}\right)$ up to 10 levels for signal (Set-3) shown in Figure 12b.

Figure 14 shows the signal gathered and the denoised signals obtained by various methods. Recovery of PD pulses and noise rejection by the methods $A$ and $B$ is shown in Figures $14 \mathrm{c}$ and $14 \mathrm{~d}$, respectively. Though, these filtering methods result in appreciable reduction in noise level $(-5.4 \mathrm{~dB}$ and $-9.2 \mathrm{~dB}$ respectively), but it is still hard to make out the presence of corona pulses by visual inspection of the denoised signal plots. Further, it is seen that, only method $\mathrm{C}$ was effective in successfully recovering the corona pulses (Figure 14e). Noise reduction in this case was about $-5.7 \mathrm{~dB}$. In this case "Daubechies 20" was used as the mother wavelet and the signal was decomposed up to 10 levels (see Figure 15). The denoised signal was obtained by adding reconstructed detail components at levels $5,6,7,8$ and 9 only. This example shows that the method is also capable of effectively denoising when DSI, low frequency interference are simultaneously present along with the PD pulses. All three types of interferences have successfully been suppressed by the wavelet method.

From the results obtained by processing these practical and excessively noisy PD signals, it emerges that wavelet based denoising technique definitely possesses superiority over the other two methods in rejecting noise and interference. The proposed wavelet method has advantages over the conventional digital filtering methods. Among others, it includes existence of a fast algorithm (it takes about $1.07 \mathrm{~s}$ to process $200 \mathrm{k}$ samples by method $\mathrm{C}$ whereas, methods $\mathrm{A}$ and $\mathrm{B}$ take $0.88 \mathrm{~s}$ and $1.02 \mathrm{~s}$, respec- 

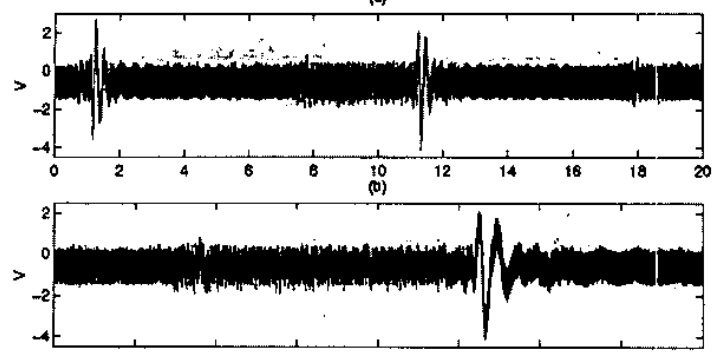

(c)

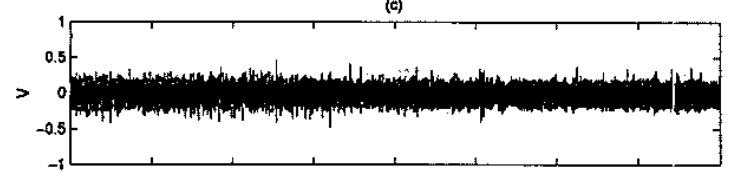

(d)
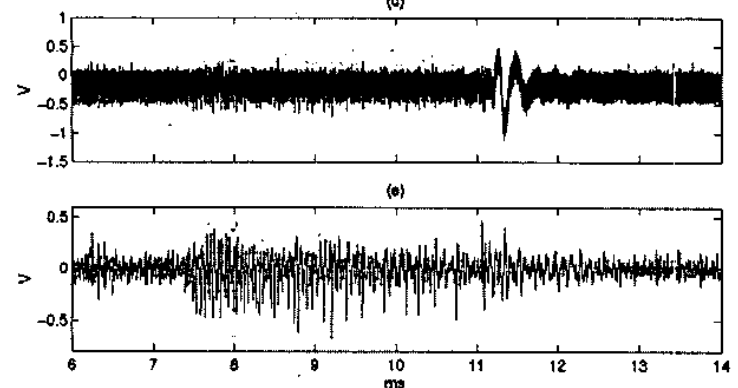

Figure 14. Results of processing practical signal Set-4. a, Acquired signal; b, Portion of (a) processed; c, Method A; d, Method B; e, Method C.

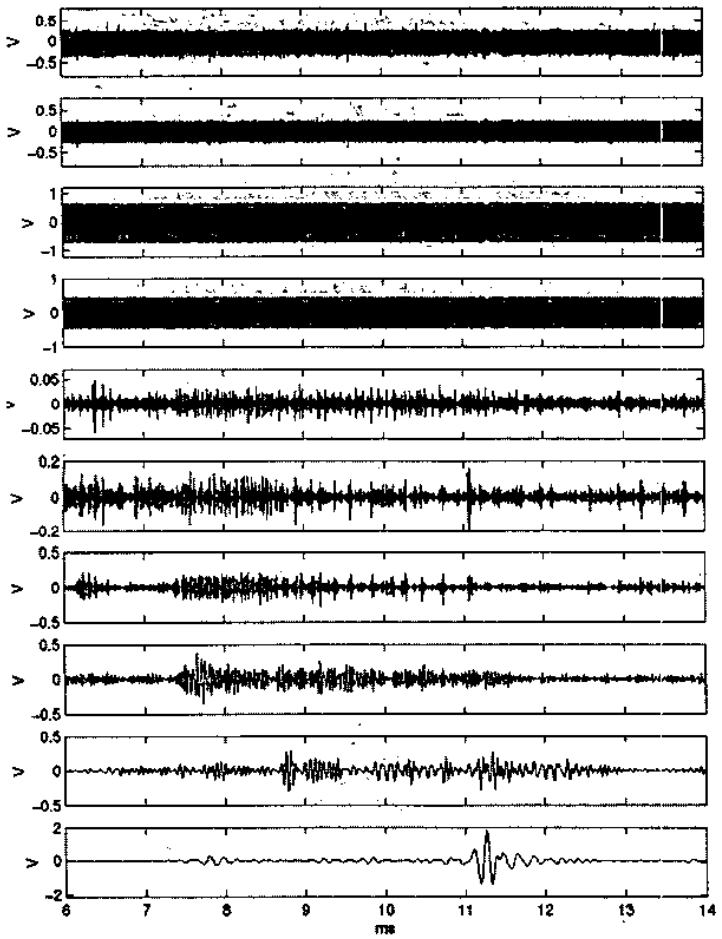

Figure 15. Reconstructed detail components $\left(D_{1}-D_{10}\right)$ up to 10 levels for signal (Set-4) shown in Figure 14b. tively on an IBM Workstation P200), ability to achieve a finer level of filtering even when the desired and interfering frequencies are overlapping and very close (both in time and frequency), and results in a low distortion of the extracted PD pulses. Perhaps, the method's only limitation is related to the choice of the mother wavelet, its number of coefficients, number of decomposition levels, etc. However, in spite of this inherent shortcoming, it has been clearly demonstrated how the potential of the wavelet method can be exploited to achieve required goals, that have not been so far achievable with earlier approaches.

\section{CONCLUSION}

$\mathrm{R}$ ELIABILITY of PD data gathered during on-line and/or on-site PD measurements is strongly influenced by external interferences. This paper presents results of the application of a novel, semi-automatic and empirical wavelet method (based on multi-resolution signal decomposition) for extracting PD pulses buried in very high levels of noise and interferences, often encountered during on-line and on-site PD tests and measurements. Further, these results are also compared with those obtained from digital filtering approaches, namely, the FIR filtering method and IIR-notch filter method, which have been reported to be suitable for this task. Analysis of the results for both simulated and practical PD waveforms reveal that, the multi-resolution analysis method has distinct advantages over the other methods. Processing of Set-1 and Set-2 signals revealed the effectiveness of the proposed method in obtaining a fine separation of the interfering frequency from the main frequency of the PD pulse, when the two frequencies are very nearly the same. Whereas, the other two methods fail to do so. Further, from processing signal Set-3, it can be concluded that, wavelet method can easily handle critical cases, where the PD pulses are superimposed (and overlap-in-time) by pulsive interference of comparable magnitude. Lastly, processing of Set- 4 shows the superiority of the wavelet based method in simultaneously removing random, DSI and pulsive interferences, over other digital methods. Thus, the proposed wavelet method was shown to be successful in rejecting all the three types of interferences, viz., random, discrete spectral and pulsive, and any combination of these occurring at the same time; rejection of the last two of these is known to be the most troublesome. Ability to process such difficult examples is being reported for the first time. In addition to achieving acceptable levels of noise suppression, another notable feature of the proposed method was that, it resulted in a minimum distortion of the PD pulses, compared to the other methods. In final conclusion, the proposed wavelet-based denoising technique was shown to be superior, with abilities to denoise even excessively noisy signals that contain various interferences (and occurring simultaneously and overlapping-intime with $\mathrm{PD}$ pulses). 


\section{REFERENCES}

[1] G. C. Stone, "Partial Discharge-Part VII; Practical Techniques for Measuring PD in Operating Equipments", IEEE Electrical Insulation Magazine, Vol. 4, No. 4, pp. 9-16. 1991.

[2] P. Osvath, W. Zaengl and H. J. Weber, "Measurement of Partial Discharge: Problems and How They Can be Solved With Flexible Measuring Systems", Tettex Instruments Bulletin SEV/VSE 76 (1985) 19, ISSN 036-1321.

[3] F. H. Kreuger, Partial Discharge Detection in High Voltage Equipment, Butterworths \& Co. Ltd, 1989.

[4] K. Feser, G. Konig, J. Ott and P. Setiz, "An Adaptive Filter Algorithm for On-site Partial Discharge Measurements", IEEE Intern. Sympos. Electrical Insulation, Boston, USA, pp. 242-245, 1988.

[5] G. Konig and K. Feser, "A New Digital Filter to Reduce Periodic Noise in Partial Discharge Measurements", 6th Intern Sympos. HV Engineering, New Orleans, USA, Paper 43.10, pp. 1-4, 1989.

[6] U. Kopf and K. Feser, "Rejection of Narrow-band Noise and Repetitive Pulses in On-site PD Measurements", IEEE Trans. DEI, Vol. 2, pp. 1180-1191, 1995.

[7] H. Borsi and M. Hartje, "New Methods to Reduce Disturbance Influences on the in situ-Partial Discharge (PD) Measurements and Monitoring", 6th Intern. Sympos. HV Engineering, New Orleans, USA, Paper 15.10, 1989.

[8] S. Zaman, D. Zhu, X. Jin and K. Tan, "An Adaptive Digital System to Reduce Periodical Noise in On-line Partial Discharge Monitoring", 8th Intern. Sympos. HV Engineering, Yokohama, Japan, Paper 63.01, pp. 77-80, 1993.

[9] H. Borsi, E. Gockenbach and U. Schichler, "PD Measurements Under Noisy Condition-Possibilities and Limits of Digital Noise Rejection", 8th Intern. Sympos. HV Engineering, Yokohama, Japan, paper 60.05 , pp. 17-20, 1993.

[10] Q. Su, "Application of Digital Signal Processing Techniques for Noise Suppression in PD Measurements", IEEE 4th Intern. Conference on Properties and Applications of Dielectric Materials (ICPADM), Brisbane, Australia, pp. 602-605, 1994.

[11] V. Nagesh and B. I. Gururaj, "Evaluation of Digital Filter for Rejecting Discrete Spectral Interferences in On-site PD Measurements", IEEE Trans. Electrical Insulation, Vol. 28, pp $73-84,1993$.

[12] M. Massanori, T. Okana, S. Nishimoto, I. Kitani and K. Arli, "Study on Application of Wavelet Analysis for Degradation Diagnosis of Partial Discharge in Void", IEEE 5th Intern. Conf. on Conduction and Breakdown in Solid Dielectrics, Leicester, UK, pp. 371-375, 1995.

[13] S. Fujimori, T. Endoh, K. Mitsuboshi, K. Ishiwata, M. Akasaka and S. Hirute, "Wavelet Analysis of Tree Development", IEEE 5th Intern. Conference on Conduction and Breakdown in Solid Dielectrics, Leicester, UK, pp. 376-380, 1995.

[14] K. Arli, M. Shibahara and M. Fujii, "Separation of Noise from Partial Discharge Signals by Wavelets", IEEE 5th International Conference on Properties and Applications of Dielectric Materials (ICPADM), Seoul, Korea, pp. 232-235, 1997.

[15] Y. Quan, N. Gao, G. Zhang and Z. Yan, "Wavelet Transform Applying in Partial Discharge Measurements", IEEE Intern.
Sympos. Electrical Insulation, Arlington, Virginia, USA, pp. 428-431, 1998.

[16] M. Florkowski, "Wavelet Based Partial Discharge Image Denoising", 11th International Sympos. HV Engineering, London, U.K., pp. 5.21.S8-5.24.S8, 1999.

[17] H. Wang, K. Tan and D. Zhu, "Extraction of Partial Discharge Signals Using Wavelet Transform", IEEE 5th Intern. Conference on Properties and Applications of Dielectric Materials (ICPADM), Seoul, Korea, pp. 322-325, 1997.

[18] I. Shim, J. J. Soraghan and W. H. Siew, "Detection of PD Utilizing Digital Signal Processing Methods, Part 3: Open-Loop Noise Reduction", IEEE Electrical Insulation Magazine, Vol. 17, No. 1, pp. 6-11, 1997.

[19] I. Shim, J. J. Soraghan and W. H. Siew, "A Noise Reduction Technique for On-line Detection and Location of Partial Discharges in High Voltage Cable Networks", Measurement Science Technology, Vol. 11, pp. 1708-1713, 2000.

[20] R. K. Young, Wavelet Theory and its Applications, Pennsylvania State University, Kluwer Academic Publishers, 1993.

[21] A. V. Oppenheim, R. W. Schafer and J. R. Buck, Discrete-Time Signal Processing, Prentice Hall, New Jersey, 1998.

[22] G. Zingales, "The Requirements of a PD Measuring System Analyzed in Time Domain," IEEE Trans. DEI, Vol.7, pp. 2-5, 2000 .

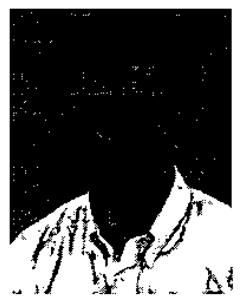

L. Satish (SM '02) was born in 1964. He received the $\mathrm{Ph} . \mathrm{D}$. degree from the Indian Institute of Science (IISc), Bangalore, in 1993. He was a Postdoctoral Fellow at ETH, Zurich, Switzerland and also worked for Tettex Instruments AG, from 1993 to 1995. In 1995, he joined the Department of High Voltage Engineering, Indian Institute of Science, where he is currently an Associate cation of signal processing to HV impulse testing and diagnostics, transfer function of transformer windings, calibration of digitizers, partial discharge measurements and pattern recognition. He was also involved with the present revision of IEC 60270 and IEC 61083-1. He was a Visiting Researcher at the HV Institute, Helsinki University of Technology, Helsinki, Finland, during the summer of 1998. Dr. Satish is a member of TF 1.1 of the CIGRE WG 33-03.

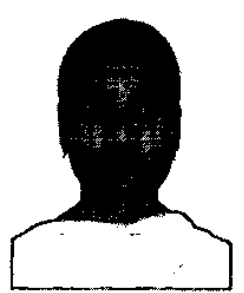

Nazneen Banu was born on May 25, 1977. She received the B.E. (Hon.) degree in Electrical Engineering from Maharishi Dayanand University, Rohtak, India, in 1999, and the M.Sc. (Eng.) degree from the Indian Institute of Science, Bangalore, in 2002. Her current research interest is in the application of signal processing methods for denoising partial discharge signals. Since May 2002, she has been an Assistant Executive Engineer in the Department of Military Engineering Services, Ministry of Defense, India. 